\title{
A Minimum Pointing Error Based Beam-Tracking Algorithm for Multi-beam GEO Satellite Antenna
}

\author{
Jian LI \\ Satellite and Wireless Communication Laboratory, School \\ of Electronics Engineering and Computer Science \\ Peking University \\ Beijing, China \\ e-mail: lixiaojian@pku.edu.cn
}

\author{
$\mathrm{Xu}$ TAN \\ Satellite and Wireless Communication Laboratory, School \\ of Electronics Engineering and Computer Science \\ Peking University \\ Beijing, China \\ Yong LUO \\ Satellite and Wireless Communication Laboratory, School \\ of Electronics Engineering and Computer Science \\ Peking University \\ Beijing, China
}

\author{
Jianjun WU \\ Satellite and Wireless Communication Laboratory, School of Electronics Engineering and Computer Science \\ Peking University \\ Beijing, China \\ e-mail: just@pku.edu.cn
}

\begin{abstract}
In this paper, the traditional LMS algorithm is extended to the real-time multi-reference point algorithm. The adaptive tracking beam is obtained by the mean square error and the minimum optimization criterion. Finally, this paper presents a switching proposal of the tracking beam. In order to better realize the effect of the proposal, the sum of minimum mean square error criterion is discussed, and the central optimal principle is proposed to choose feed combination. The central optimal principle proposed in this paper can select a more suitable combination of feeds to form the tracking beam, and then obtain a better adaptive tracking effect.
\end{abstract}

Keywords-multi-beam satellite antenna; real-time multi-reference LMS algorithm; tracking beamforming technology; beam switching; feed selection; central optimal principle

\section{INTRODUCTION}

In the field of satellite communication system, research on multi-beam Satellite Antenna has been gradually changed from traditional fixed multi-beam networking to the user-centric service mechanism. The user-centric service mechanism needs flexible configuration ability within the dynamic resources. The tracking beam-forming technology based on parabolic reflector multi-beam satellite is an effective way to solve this problem.
The system involved in this paper uses a single parabolic reflector antenna, consistent with the common system, the feed array of the system is placed in the position of focus, and generating synthesized beams by the enhancing beam forming method to cover of the target area. In the same time, a real-time multi-reference LMS algorithm is obtained by extending the traditional LMS algorithm. And the real-time beam-forming weighting coefficients are calculated by the mean square error and the minimum optimization criterion. In addition, a new method of beam switching and feed selecting scheme is proposed to ensure that the difference of signal quality between the synthetic beam at the target angle and the centre of the target beam is as small as possible.

\section{MULTI - BEAM SATELLITE SYSTEM MODEL}

The applications of multi-beam satellite mobile communication system in the field of information technology are much more frequent than ever before. The multi-beam satellite mobile communication system may form a lot of beam cells and serve the target area through them. The feed array is the core part of the whole system because it's the emission source of the system. Usually, it is placed in the focal position, so the entire arrays are not all in focus, almost all the feeds are partial focus feed, these different positioned feeds are able to produce different directional beams, and then cover the target areas. In general, the schematic diagram of the overall system is shown below: 


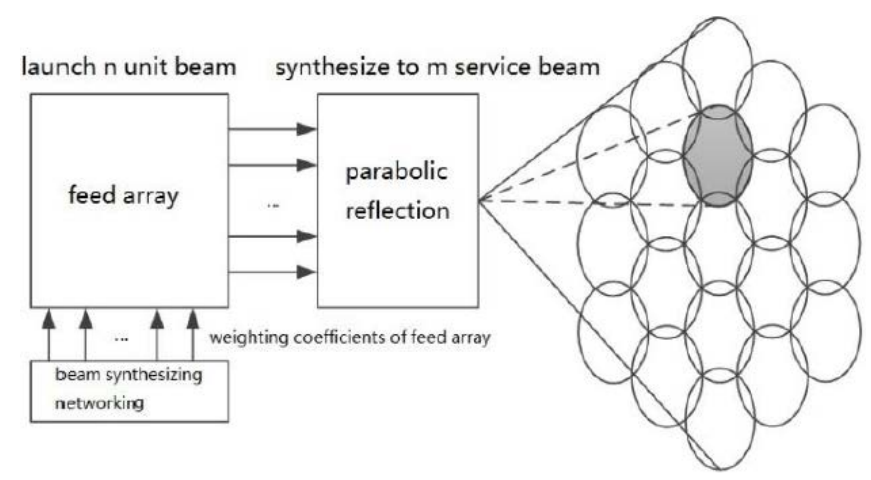

Figure 1. Multi - beam system diagram

The beamforming network adjusts the weighting coefficients of the respective feeds so that the single feed beam can be effectively combined to obtain the target synthesized beam. The beam synthesis network is illustrated as follows:

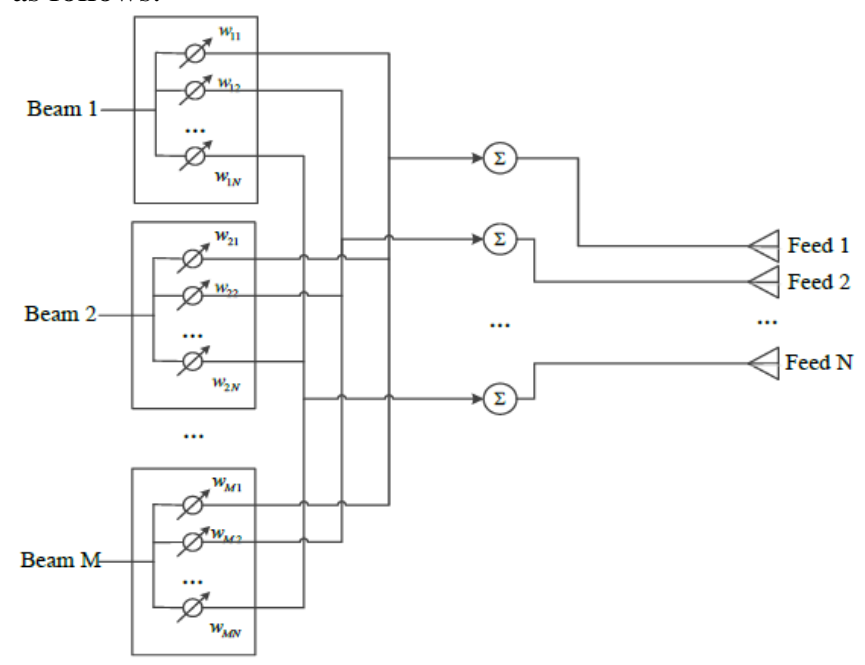

Figure 2. Beam network synthesis diagram

It can be seen that each target composite beam corresponds to a set of weighting coefficients. Among them, $w_{m n}$ is plural, which indicates the effect on amplitude and phase. The physical meaning is the weighting coefficient corresponding to the nth feed when the mth target beam is synthesized. The synthesized beam's expression:

$$
E_{m}(\Phi, \Psi)=\sum_{n=1}^{N} w_{m n} \varepsilon_{n}(\Phi, \Psi)
$$

The tracing scene of the tracking beam is set as follows: the ground user terminal has GPS module, and the tracking beam is formed for a particular user in the case of reserving the primitive networking mode of the ground for initial access. In this case, the gateway station can obtain the location information of the user through GPS coordinates, therefore, the key point of beam forming is to adjust the tracking beam forming factor, to ensure that the tracking beam centre can correctly point to the target user or user group; and because of the user's mobility, the time-varying characteristics and computational speed are required in tracking beam forming algorithm.

Firstly, let the radiation field of the mth synthetic beam at time $\mathrm{t}$ is $E_{m}(\Phi, \Psi, t)$, here take $\mathrm{K}$ reference points, $\hat{d}=\left[E_{m}\left(\Phi_{1}, \Psi_{1}, \mathrm{t}\right) \quad E_{m}\left(\Phi_{2}, \Psi_{2}, \mathrm{t}\right) \quad \ldots \quad E_{m}\left(\Phi_{K}, \Psi_{K}, \mathrm{t}\right)\right]$, the initial signal matrix is:

$$
X=\left(\begin{array}{ccc}
\varepsilon_{1}\left(\Phi_{1}, \Psi_{1}, \mathrm{t}\right) & \ldots & \varepsilon_{1}\left(\Phi_{K}, \Psi_{K}, \mathrm{t}\right) \\
\vdots & \ddots & \vdots \\
\varepsilon_{N}\left(\Phi_{1}, \Psi_{1}, \mathrm{t}\right) & \cdots & \varepsilon_{N}\left(\Phi_{K}, \Psi_{K}, \mathrm{t}\right)
\end{array}\right)
$$

Set the feed weighting factor vector $\hat{w}(\mathrm{t})=\left[w_{1}(\mathrm{t}), w_{2}(\mathrm{t}), \ldots, w_{N}(\mathrm{t})\right]$, get the synthetic sequence $\hat{y}(\mathrm{t})=\hat{w}(\mathrm{t}) X(\mathrm{t}) \quad$, at this point the error is $\hat{e}(\mathrm{t})=\hat{d}(\mathrm{t})-\hat{y}(\mathrm{t})$, so the mean square error $\hat{\xi}(\mathrm{t})=|\hat{e}(\mathrm{t})|^{2}$.

Because of the multi-reference point LMS algorithm, we extends the criterion to the overall mean square error, objective function: $\min \left\{\xi(\mathrm{t})=\sum \xi_{k}(\mathrm{t})\right\}$.

Since the direction of the gradient changes the fastest in the positive, then the negative gradient corresponds to the direction in which this function decreases the fastest. Therefore, the steepest descent method is to make the weighting coefficient of iterative calculation change in the direction of negative gradient, thereby the solution is obtained after several rounds of computation.

$$
w_{k+1}(\mathrm{t})=w_{k}(\mathrm{t})+\mu\left[-\nabla_{w} f\left(w_{k}(\mathrm{t})\right)\right]
$$

Among them, $\mu$ is step value, $f(w(t))$ is a function of $w(\mathrm{t}), \nabla_{w} f\left(w_{k}(\mathrm{t})\right)$ is the gradient value at $w_{k}(\mathrm{t})$. The specific derivation of the algorithm is as follows:

For any mean square error $\xi_{k}(\mathrm{t})$, take the gradient:

\section{TRACKING BEAMFORMING TECHNOLOGY}

\section{A. Tracking Beam Synthesis Based on Extended LMS Algorithm}




$$
\begin{aligned}
\nabla_{\hat{w}} \xi_{k} & (\mathrm{t})=\nabla_{\hat{w}}\left|d_{k}(\mathrm{t})-\hat{w}(\mathrm{t}) X(k, \mathrm{t},:)^{T}\right|^{2} \\
= & \nabla_{\hat{w}}\left\{\left[d_{k}(\mathrm{t})-\hat{w}(\mathrm{t}) X(k, \mathrm{t},:)^{T}\right]\left[d_{k}(\mathrm{t})-\hat{w}(\mathrm{t}) X(k, \mathrm{t},:)^{T}\right]^{*}\right\} \\
= & \nabla_{\hat{w}}\left[\left|d_{k}(\mathrm{t})\right|^{2}-d_{k}(\mathrm{t}) X(k, \mathrm{t},:)^{H} \hat{w}^{*}(\mathrm{t})-\hat{w}(\mathrm{t}) X(k, \mathrm{t},:)^{T} d_{k}^{*}(\mathrm{t})+\hat{w}(\mathrm{t}) X(k, \mathrm{t},:)^{T} X(k, \mathrm{t},::)^{H} \hat{w}^{*}(\mathrm{t})\right] \\
= & -2\left[X(k, \mathrm{t},:)^{T} d_{k}^{*}(\mathrm{t})-X(k, \mathrm{t},:)^{T} X(k, \mathrm{t}:)^{H} \hat{w}^{*}(\mathrm{t})\right] \\
= & -2 X(k, \mathrm{t},:)^{T}\left[d_{k}(\mathrm{t})-y_{k}(\mathrm{t})\right]^{*} \\
= & -2 e_{k}^{*} X(k, \mathrm{t},:)^{T}
\end{aligned}
$$

Then

Get

$$
\nabla_{\hat{w}} \xi(\mathrm{t})=\nabla_{\hat{w}} \sum_{k=1}^{K} \xi_{k}(\mathrm{t})=\sum_{k=1}^{K} \nabla_{\hat{w}} \xi_{k}(\mathrm{t})=\sum_{k=1}^{K}-2 e_{k}^{*} X(k, \mathrm{t},:)^{T}=-2 \hat{e}^{*} X(\mathrm{t})^{T}
$$

$$
\sum_{k=1}^{K}\left[X(k, \mathrm{t},:)^{T} d_{k}^{*}(\mathrm{t})-X(k, \mathrm{t},:)^{T} X(k, \mathrm{t},:)^{H} \hat{w}(\mathrm{t})^{*}\right]=0
$$

Therefore

$$
\hat{w}(n+1, t)=\hat{w}(n, t)+2 \mu \hat{e}^{*} X(\mathrm{t})^{T}
$$

The formula above is the multi-reference time-varying LMS iterative formula.

Sum of the mean square error

$$
\xi(\mathrm{t})=\sum_{k=1}^{K}\left|d_{k}(\mathrm{t})-\hat{w}(\mathrm{t}) X(k, \mathrm{t},:)^{T}\right|^{2}=\sum_{k=1}^{K}\left(\left|d_{k}(\mathrm{t})\right|^{2}-2 \hat{w}(\mathrm{t}) r_{x d k}(\mathrm{t})+\hat{w}(\mathrm{t}) R_{x x k}(\mathrm{t}) \hat{w}(\mathrm{t})^{H}\right)
$$

Among them

$$
\begin{gathered}
r_{x d k}(\mathrm{t})=d_{k}(\mathrm{t}) X(k, \mathrm{t},:)^{T} \\
R_{x x k}(\mathrm{t})=X(k, \mathrm{t},:)^{T} X(k, \mathrm{t},:)^{*}
\end{gathered}
$$

The steepest descent method can obtain the minimum of the sum of mean squared error by iteration, the extreme value is obtained by seeking derivative in this section,

Make

$$
\nabla_{\hat{w}} \xi(\mathrm{t})=0
$$

Finally

$$
\hat{w}_{\text {opt }}(\mathrm{t})=\left(\sum_{k=1}^{K} R_{x x k}(\mathrm{t})\right)^{-1} \sum_{k=1}^{K} r_{x d k}(\mathrm{t})
$$

The formula above is the Wiener solution for this problem.

\section{B. Beam Switching and Feed Selection}

When the users or the terminals moves across the area, the corresponding satellite mobile communication system dispatches another beam cell to them. In this paper, the decision threshold of the beam switching is a $-1 \mathrm{~dB}$ loop outside the current tracking beam centre. The sum of minimum mean square error criterion is a simple and intuitive method whose index function is $\xi=\sum \xi_{k}$, the final objective function is $i=\min _{i}(\xi(\mathrm{i}))=\min _{i}\left(\sum \xi_{k}(\mathrm{i})\right)$.

However, this criterion has a disadvantage that the centre beam in some cases. In this section, according to the requirements of the tracking beam, we design the 'central optimal principle' for the tracking beam's central signal angle and amplitude. The calculation process is as follows: of the selected beam would differ from the target service 


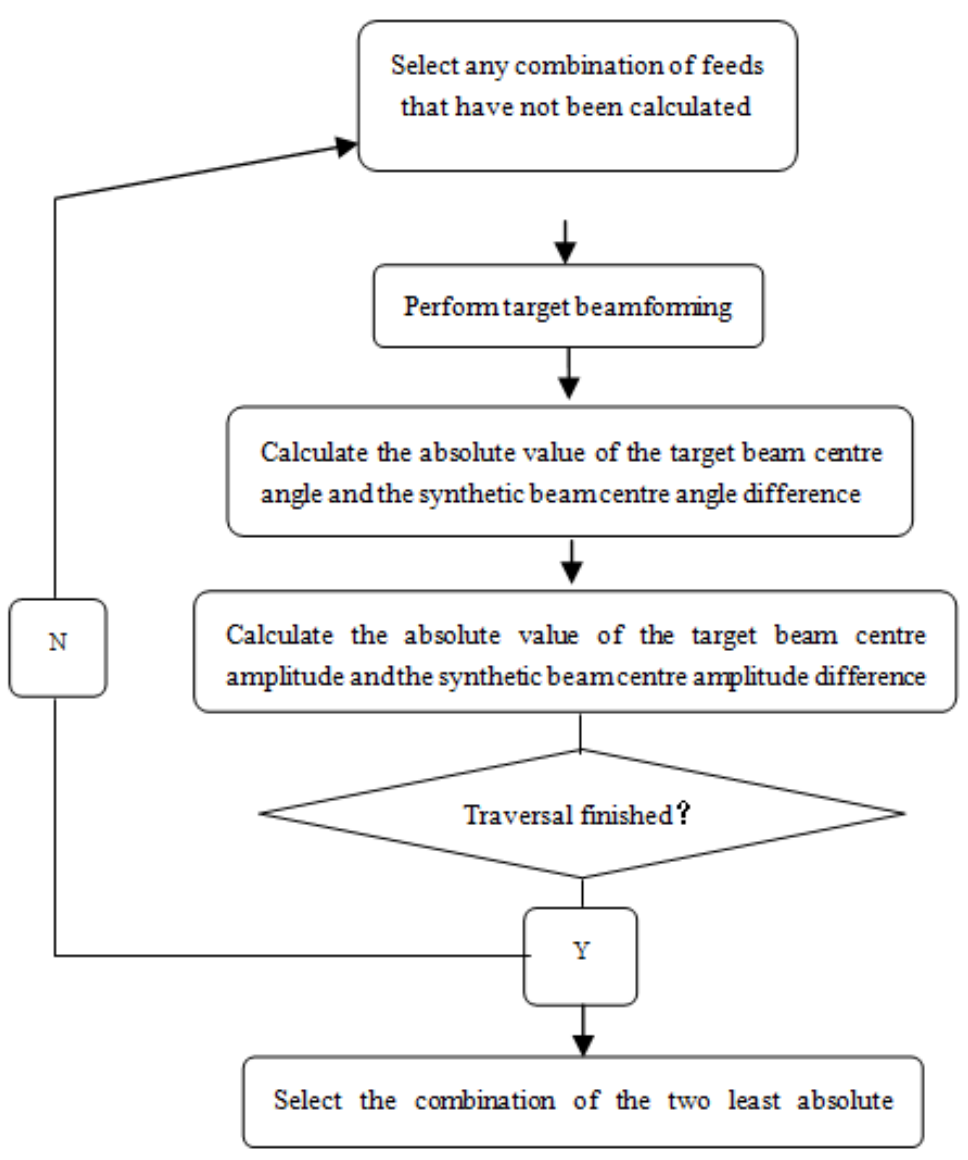

Figure 3. Calculation process of central optimal principle

The objective function of the central optimal principle is:

$$
i=\min _{i}(\theta(\mathrm{i})+S(\mathrm{i}))=\min _{i}\left(\left|\theta_{\text {target }}(\mathrm{i})-\theta_{\text {beamformin } g}(\mathrm{i})\right|+\left|\mathrm{S}_{\text {target }}(\mathrm{i})-\mathrm{S}_{\text {beamforming }}(\mathrm{i})\right|\right)
$$

Where $\mathrm{i}$ is the feed combination number, $\theta_{t \text { arg } e t}$ represents the angle of the target beam centre, $\mathrm{S}_{\text {beamformin } g}$ represents the angle of the synthetic beam centre, $\theta_{t \text { arg et }}$ represents the beam amplitude at the centre $\theta_{\text {target }}$ of the target beam, $\mathrm{S}_{\text {beamforming }}$ represents the beam amplitude at $\theta_{\text {target }}$ in the synthesized beam. The principle of the objective function is: Ensure that the difference of angle between the synthetic beam centre and the target beam centre is as small as possible; the difference of the signal quality between the synthesized beam at the target angle (the angle of the target beam centre) and the signal of the target beam centre is as small as possible.

\section{NUMERICAL CALCULATION}

Firstly, we use 7 feed sources to synthesize the tracking beam in the $0{ }^{\circ}$ direction of the centre, as shown below: 


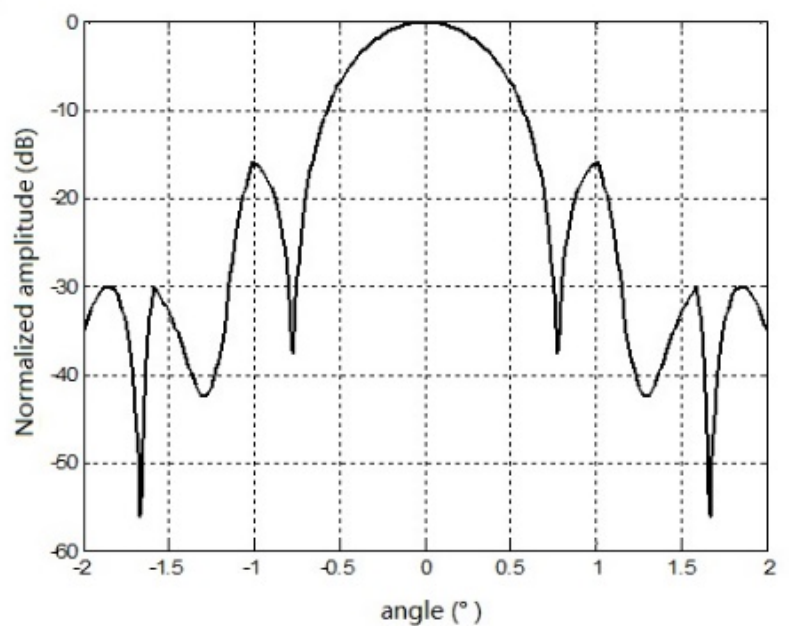

Figure 4. Target angle $0^{\circ}$ Tracking beamforming pattern

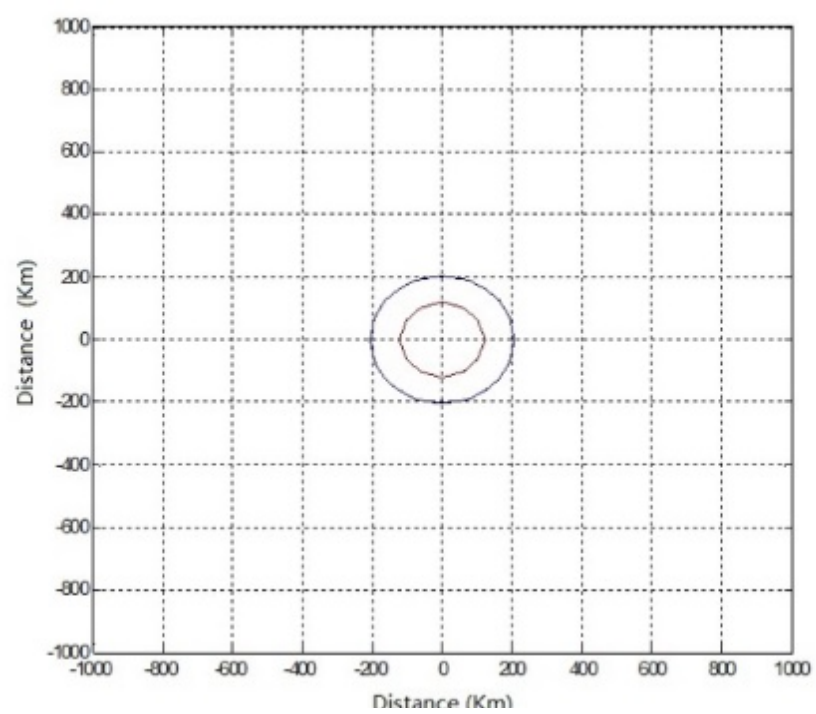

Figure 5. Target angle $0^{\circ}$ Tracking beamforming Projection map pattern

The centre of beam point to the $0{ }^{\circ}$ direction accurately, the shape and attenuation of the main lobe meets the requirements of the tracking service beam very well. The red line in the figure represents $-1 \mathrm{~dB}$ contour coil, blue line represents $-3 \mathrm{~dB}$ contour coil, $-3 \mathrm{~dB}$ circle corresponding to the ground distance of $200 \mathrm{Km},-1 \mathrm{~dB}$ circle corresponding to about $120 \mathrm{Km}$.

It can be seen that a well-formed circular beam projection is formed in the central region, that is the ground cell, the cell radius also meets the criteria. For any non-centre point beam, the numerical calculation verification is based on the adjacent cell. Take $-3 \mathrm{~dB}$ overlapping networking for example, it correspond to the ground distance of $200 \mathrm{Km}$, the cell centre distance is $\sqrt{3}$ times the cell radius, the deviation angle of the beam centre is $0.5223^{\circ}$ corresponding to the GEO satellite. The numerical results are shown below:

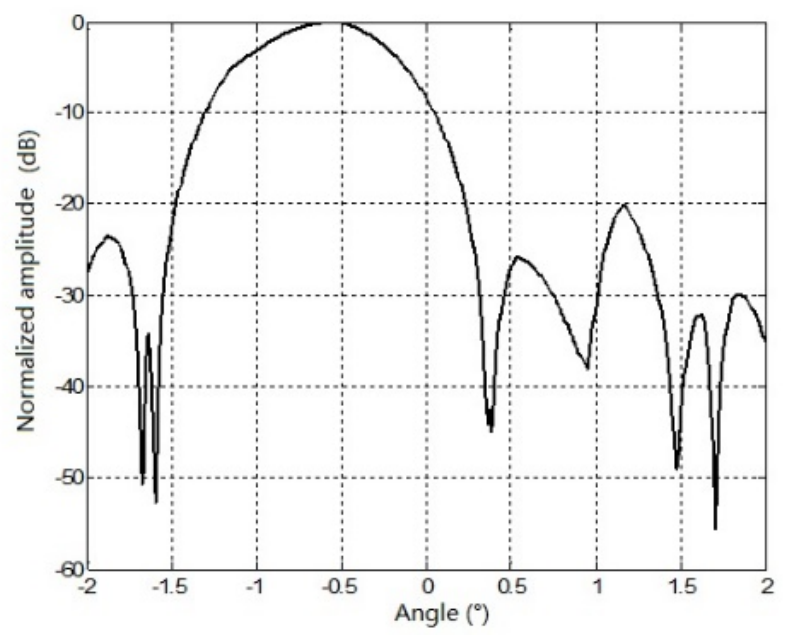

Figure 6. Target angle $0.5223^{\circ}$ tracking beamforming pattern

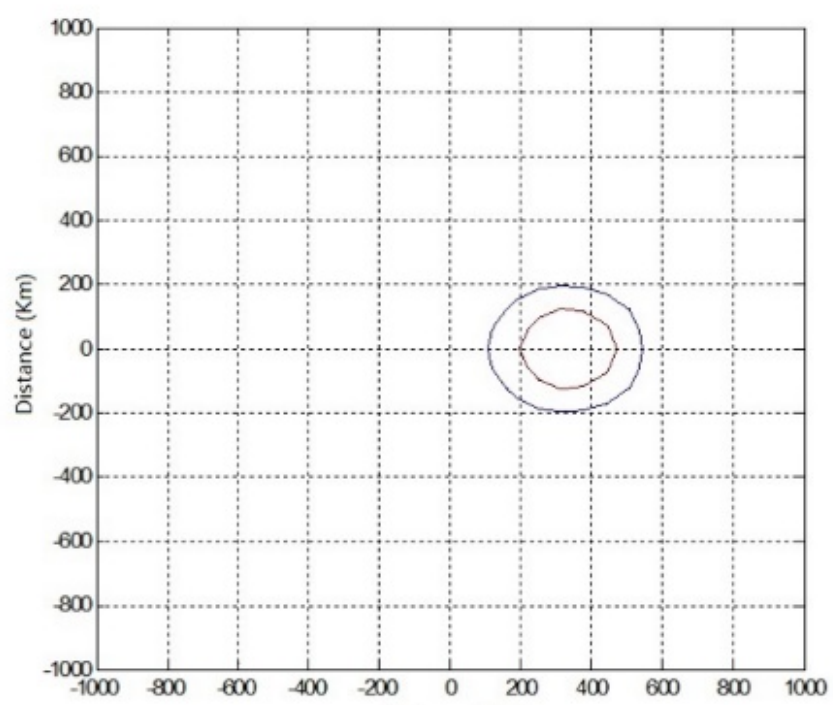

Figure 7. Target angle $0.5223^{\circ}$ tracking beamforming Projection map

The beam centre is oriented exactly at $0.5223^{\circ}$, and the shape and attenuation of the main lobe fully meet the requirements of the tracking service beam. The red line in the figure represents $-1 \mathrm{~dB}$ contour coil, blue line represents $-3 \mathrm{~dB}$ contour coil. For the service beam at the central location, the beam width of $0.5223^{\circ}$ corresponds to a position of approximately $346 \mathrm{Km}$ above the ground surface. Considering the angle to launch beams, the non-centre 
vertical projection cell will be shown like non-circular shape, the $-1 \mathrm{~dB}$ and $-3 \mathrm{~dB}$ contour coil also reached the basic goal of tracking beam forming.

\section{CONCLUSION}

For the multi-beam satellite developed in the user-centric direction, the research of tracking beam forming technology is carried out in combination with the project of Natural Science Foundation in this paper. A real-time multi-reference points LMS algorithm is obtained by extending the traditional LMS algorithm, and through the mean square error and the minimum optimization criterion, we get the tracking beam, it provides the basis for the following discussion of tracking beam applications. Through numerical analysis, the extended algorithm can easily get the weighting coefficient of the time-varying feed source, thereby we can obtain a tracking beam of arbitrary angle. In this paper, the selection and extended calculation of the LMS algorithm is correct, we can achieve the desired goal when synthesize tracking beam at any angle using the new algorithm method.

\section{ACKNOWLEDGEMENTS}

This work is supported by the National Natural Science Foundation of China (No.61371073). Corresponding author: Jianjun Wu; Phone: +86-10-62752848; Email: just@pku.edu.cn.

\section{REFERENCES}

[1] Lezhu Zhou, Dou Li, Wenjia Guo, "satellite communications multi-beam antenna review" Electronics Journal, Acta Electronica Sinica, 2001,06\#

[2] Hongfen Hu, Xiaohong Bai, "Application and Development of Multi-beam Antenna" Modern Radar, 2002,04\#

[3] Fengyi Xie, "Europe's two major satellite companies to expand Ka-band broadband market," Satellite TV \& IP Multimedia, 2013,15\#

[4] P.Angeletti, G.Gallinaro, M.Lisi and A.Vernucci, On-ground digital beamforming techniques for satallite smart antennas, ICSSC 2001

[5] Yongxuan Xiao, Yong Xue, Xiaojin Zeng. "GEO mobile communication satellite synthetic multi-beam antenna simulation analysis." Spacecraft Engineering 003 (2010): 74-79.

[6] Fletcher R, Powell M J D. A rapidly convergent descent method for minimization[J]. The Computer Journal, 1963, 6(2): 163-168. 